\title{
Face pose estimation using PCA
}

\author{
Bidyut Jyoti Saha ${ }^{*}$, Amitava Sen ${ }^{2}$ and Subnum Begum ${ }^{3}$ \\ IFS Technical Consultant, L\&T Infotech, Airoli, Mumbai, India ${ }^{1}$ \\ School of Computer Engineering, KIIT University, Bhubaneswar, India ${ }^{2}$ \\ Assistant System Engineer, TCS, Pune, India ${ }^{3}$
}

\section{(C)2016 ACCENTS}

\begin{abstract}
Face recognition has been one among the foremost attention-grabbing and vital analysis fields within the past 20 years. The explanations come back from the necessity of automatic recognitions and police work systems, the interest in human sensory system on face recognition, and also the style of human-computer interface, etc. These researches involve information and researchers from disciplines like neurobiology, psychology, laptop vision, pattern recognition, image process, and machine learning, etc. Different techniques like principal component analysis (PCA), 2PCA, kernel principal component analysis (KPCA) are used for recognition. Distinction factors like illumination, pose, and expression are degrading the accuracy of recognition. In this paper we have taken 11 poses of a given subject before recognition and performed pose estimation for identifying correct pose of image, hence increasing the accuracy of recognition of image.
\end{abstract}

\section{Keywords}

Face recognition, PCA, KPCA, Pose estimation.

\section{Introduction}

Our faces are complicated objects with options that may vary over time. However, we humans have a tendency to acknowledge faces and determine persons in an exceedingly look. Of course, our natural recognition ability extends on the far side of face recognition, we have a tendency to quickly acknowledge patterns, sound or smell, sadly, but this aptitude doesn't exist in machines, therefore there is the necessity for making intelligent autonomous machines to simulate recognition unnaturally.

Most sure-fire applications of image analysis and understanding, face recognition has recently received vital attention, particularly throughout the past few years. In pre 2000 era the emergence of face recognition is recognized in conference like international conference on audio and video-based authentication (AVBPA) [1] in 1997 and in international conference on automatic face and gesture recognition (AFGR) in 1995, systematic empirical evaluations of face recognition techniques (FRT) [1].

*Author for correspondence
To secure any gift day system biometric system were introduced and describes in [2]. Applications of face recognition describe in [3] and additionally later in application section.

Muruganantham [4] proposed a new technique for pose invariant face recognition where 9 different poses of a subject are taken into consideration. Liau et al. [5] proposed a multiview face recognition system based on Eigen face method.

In this paper 7 different poses of a subject is taken into consideration. Face recognition procedure is divided into 3 steps: face detection [4], feature extraction [6, 7] and face recognition [7]. Face recognition methods for intensity images are divided into three main categories: feature-based, holistic, hybrid approaches. During recognition we find different factors that affect recognition accuracy i.e. pose, expression, illumination etc.

Different techniques are used for recognition here we use PCA for recognition. Pose is one in all the issue that have an effect on face recognition lots. Accuracy of Face Recognition quite bated because of presence of various poses of an image. Pose estimation captures the correct pose of a picture. Pose estimation: Pose estimation is a specific task of determining the pose of an object in an image. Here 
PCA is used for Pose estimation. PCA reduced the dimensions of every mean face for the convenience of computation and storage. Feature extraction method is used for obtaining PCA projection vectors. The pose orientation of the probe image is calculable by calculating the probability estimation between the probe face and mean faces. Mean face that provides the utmost likelihood is presumably to possess an equivalent pose orientation as the probe face $[1,5]$.

PCA is amongst the foremost successful techniques that are utilized in image recognition and compression. PCA is a statistical method used for factor analysis. The purpose of PCA is to scale back the massive spatiality of the information space to the smaller intrinsic spatiality of feature space [8].

This is the case once there's a robust correlation between ascertained variables. The task of PCA includes square measure prediction, redundancy removal, feature extraction, information compression, etc. PCA may be a classical technique which works within the linear domain, applications having linear models square measure appropriate, like signal process, image process, system and management theory, communications, etc. [8].The main motive of using PCA for face recognition is to precise the massive 1-D vector of pixels made from 2-D facial image into the compact principal elements of the feature space, referred to as Eigen space projection $[5,8,4]$.

\section{Proposed algorithm}

Here we define three stage for recognition of face and also describe in Figure 1.

Stage I Creation of Data Base For Image Recognition.

Stage II Calculate Eigenvalues and Eigen faces.

Stage III Extraction PCA features and matching of Test Image with Train Database (Pose Estimation).

\section{Database creation}

In Figure 2 Creation of database for image recognition is described.

Step1: For creation of Database first we have to provide the path of the training database which is store in a variable TrainDatabasePath.

TrainDatabasePath = uigetdir(startpath, title)

Step2: Then we take single image from the given database.

Step3: After taking single image find out the size of image that is no of rows and no of columns.

[irowicol] $=$ size $(\mathrm{img})$

12
Step4: Then Reshape the image. This function reshapes all 2D images of training database into $1 \mathrm{D}$ column vector.

temp $=$ reshape $\left(\mathrm{img}{ }^{\prime}\right.$, irow $*$ icol, 1$)$

Step5: Then it puts these 1D column vectors in a row to construct 2D matrix ' $T$ ',

Step6: Repeat step 2-5 until database is not end.

Step7: Return T [] (A 2D matrix containing all 1D image vector).

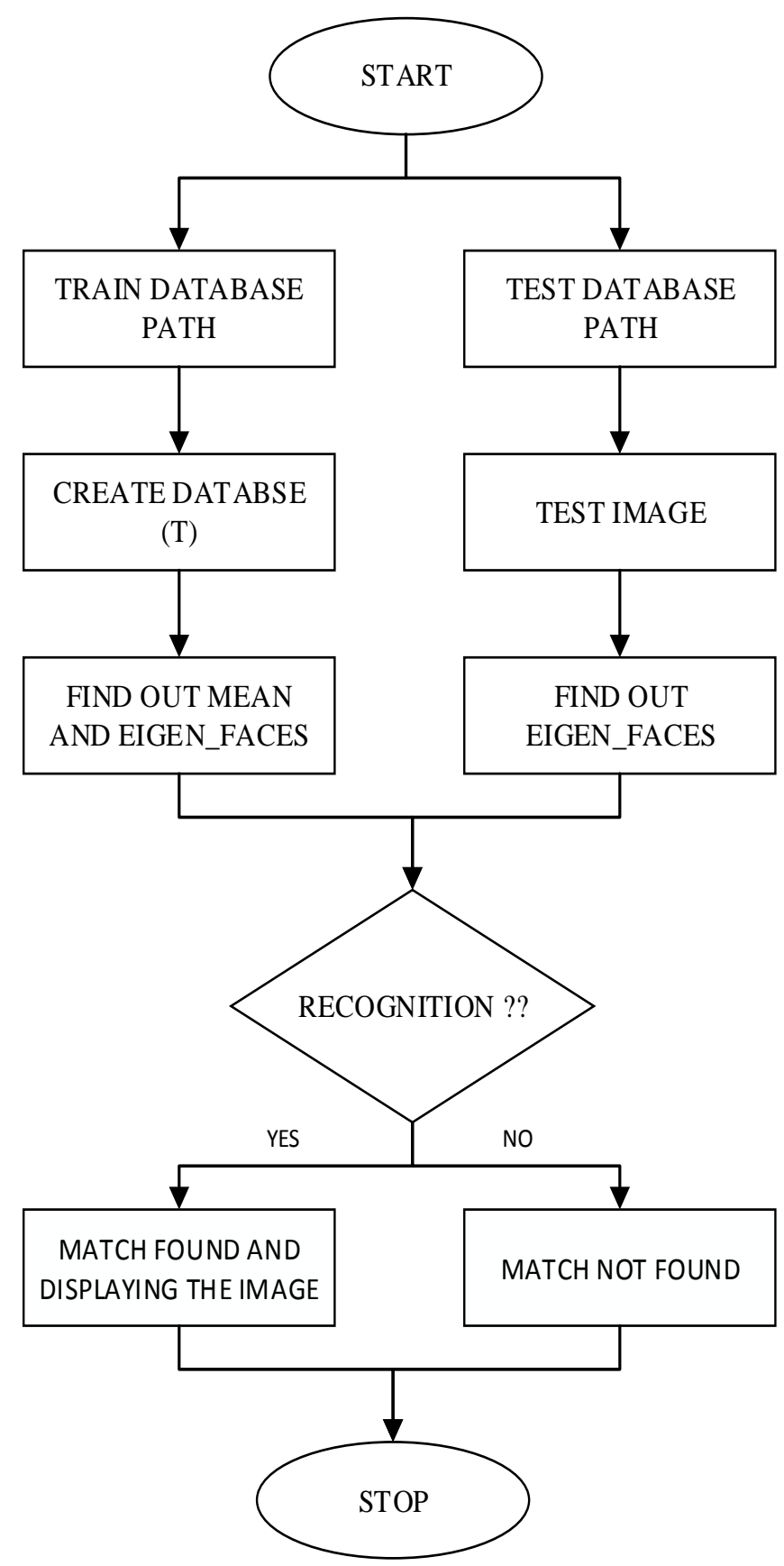

Figure 1 Face recognition steps 


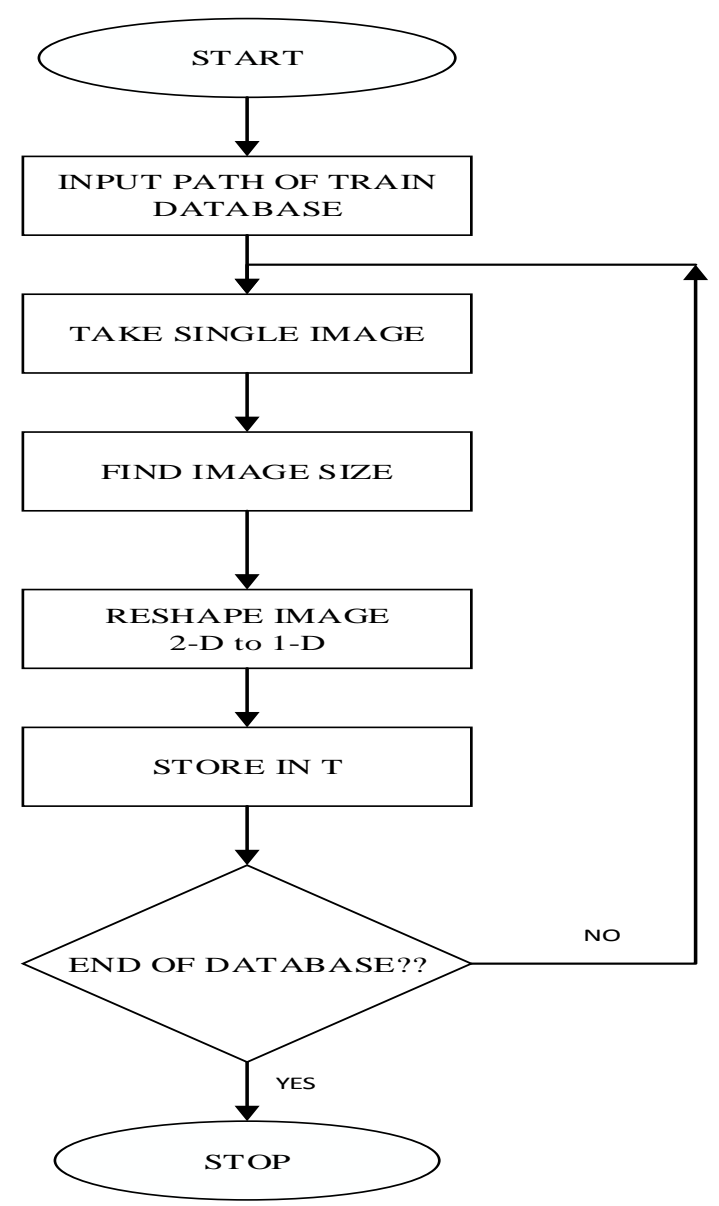

Figure 2 Creation of database for image recognition

\section{Calculation of Eigen faces}

In Figure 3 steps of eigenFaces Calculation are described.

Step1: Here we pass $\mathrm{T}$ [] as arguments which contains all training image.

Step2: Then calculating the mean from the given $T[]$. $\mathrm{M}=(1 / \mathrm{P}) * \operatorname{sum}(\mathrm{Tj}$ 's $)$

Step3: Find out deviation of each image in the training set from mean image and then store in A.

$\mathrm{Ai}=\mathrm{Ti}-\mathrm{m}$

Step4: Find out $\mathrm{L}$ and $\mathrm{V}$ from $\mathrm{A}$ where $\mathrm{L}$ is surrogate of covariance matrix and $\mathrm{V}$ is

Eigen values.

$\mathrm{L}=\mathrm{A}^{\prime} * \mathrm{~A}$

$\mathrm{V}=\operatorname{eig}(\mathrm{L})$

Step5: Then sorting and eliminating eigenvalues that is all eigenvalues of matrix $\mathrm{L}$ are $\mathrm{M}$ sorted and those who are less than 1 are eliminated.Remaining values are stored in variable leigvec.

Step6: Then find Eigenfaces.

Eigenfaces $=\mathrm{A} *$ Leigvec

Step7: Return m,Eigenfaces,A.

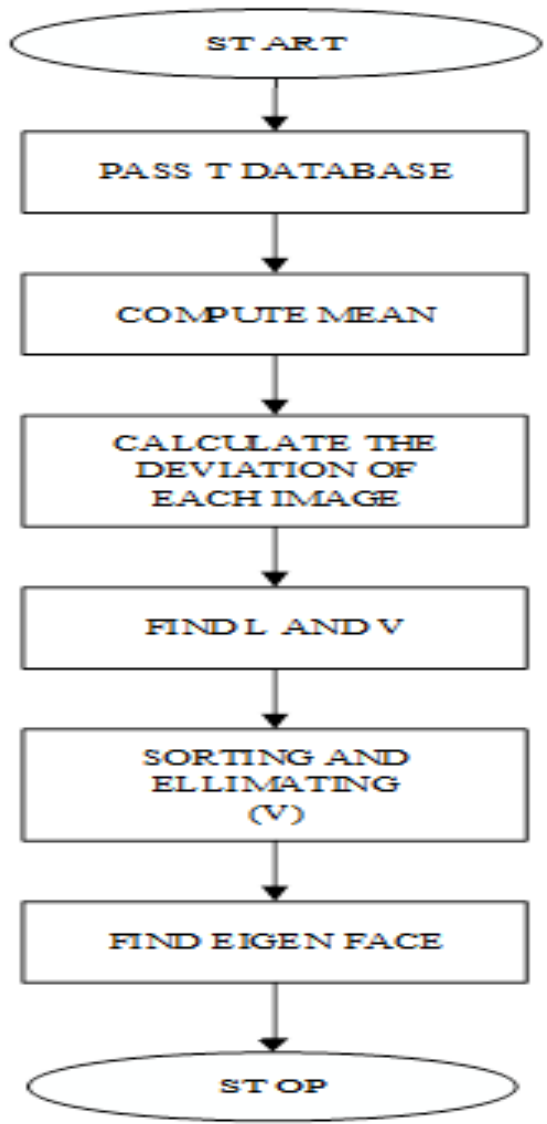

Figure 3 Calculation of Eigen faces

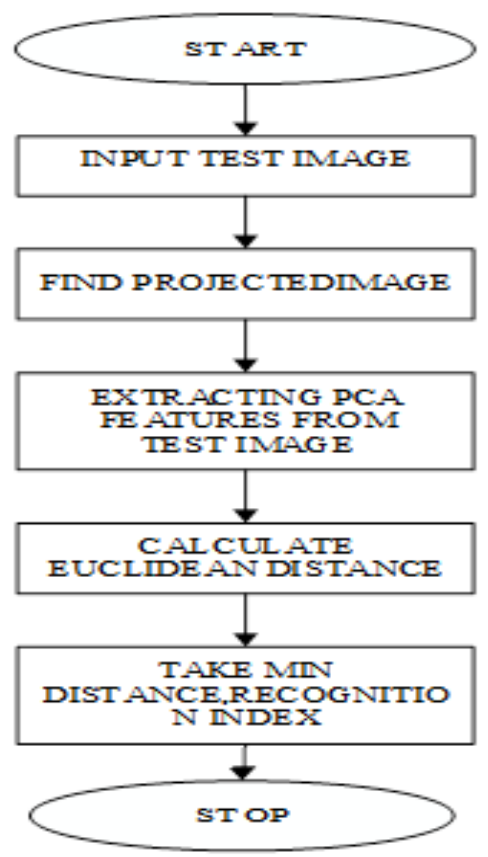

Figure 4 Pose estimation 
Bidyut Jyoti Saha et al.

5.Feature extraction and pose estimation In Figure 4 steps of Pose estimation are described. Step1: First we take TestImage, m, Eigenfaces, A as input arguments.

Step2: Find ProjectedImage that is all centered images are projected into facespace by multiplying in Eigenface and store in ProjectedImage.

ProjectedImage $=$ Eigenfaces ${ }^{\prime} * \mathrm{Ai}$

Step3: Extract PCA features from the Test Image and store in ProjectedTestImage.

inimage $=$ reshape $(. .$.

Difference $=$ inimage $-\mathrm{m}$
ProjectedTestImage $=$ Eigenfaces ${ }^{\prime} *$ Difference

Step4: Then calculating Euclidean distance between ProjectedTestImage and ProjectedImage(projection of all centered training images).

Step5: Take minimum Euclidean distance and its corresponding index and store in variable outputname.

Step6: Return outputname that is name of the recognized image in the training database.

Set of images collected for a given subject and Recognition rate are shown in Figure 5 and Figure 6.

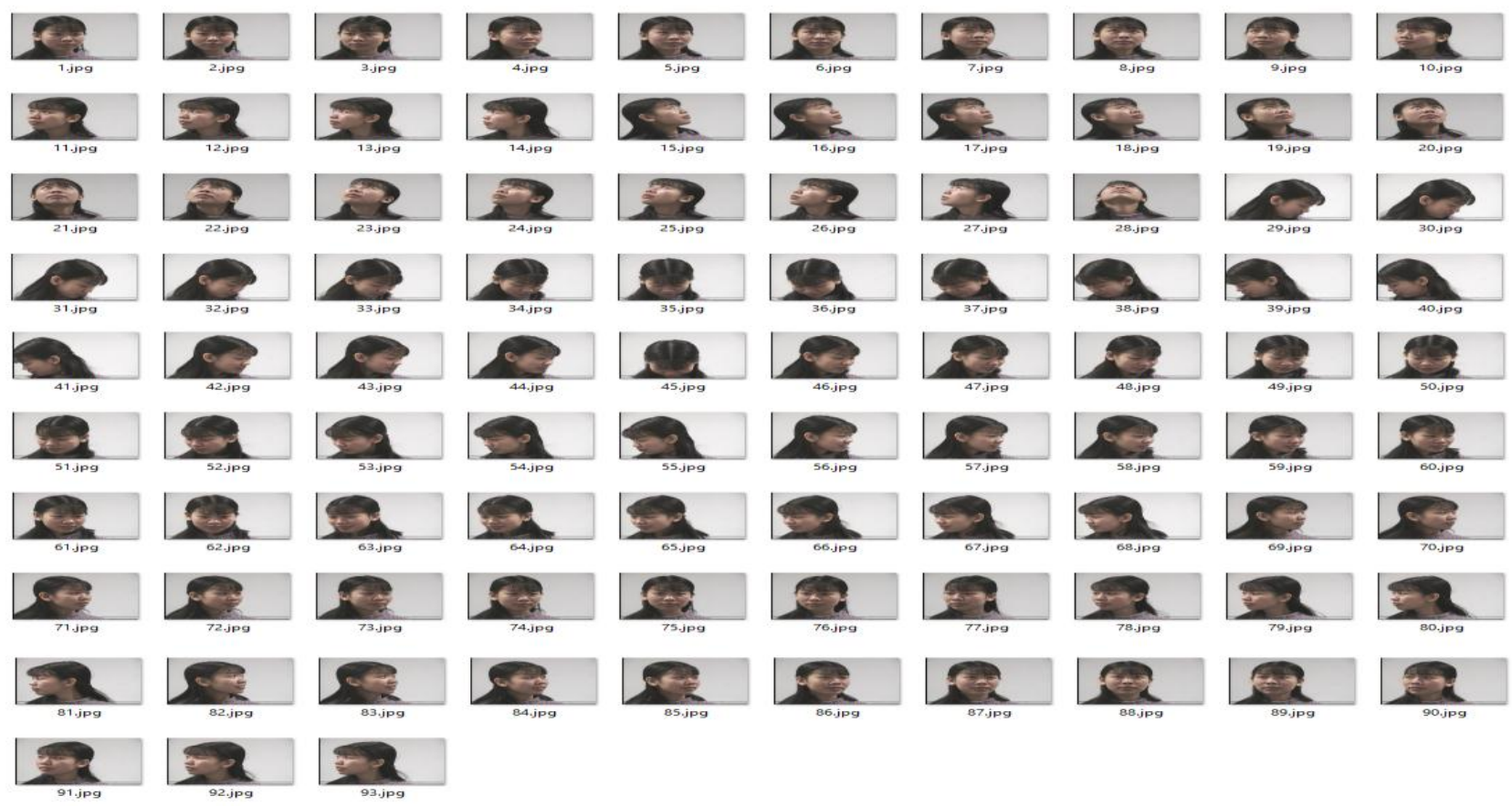

Figure 5 Typical set of images collected for a given subject

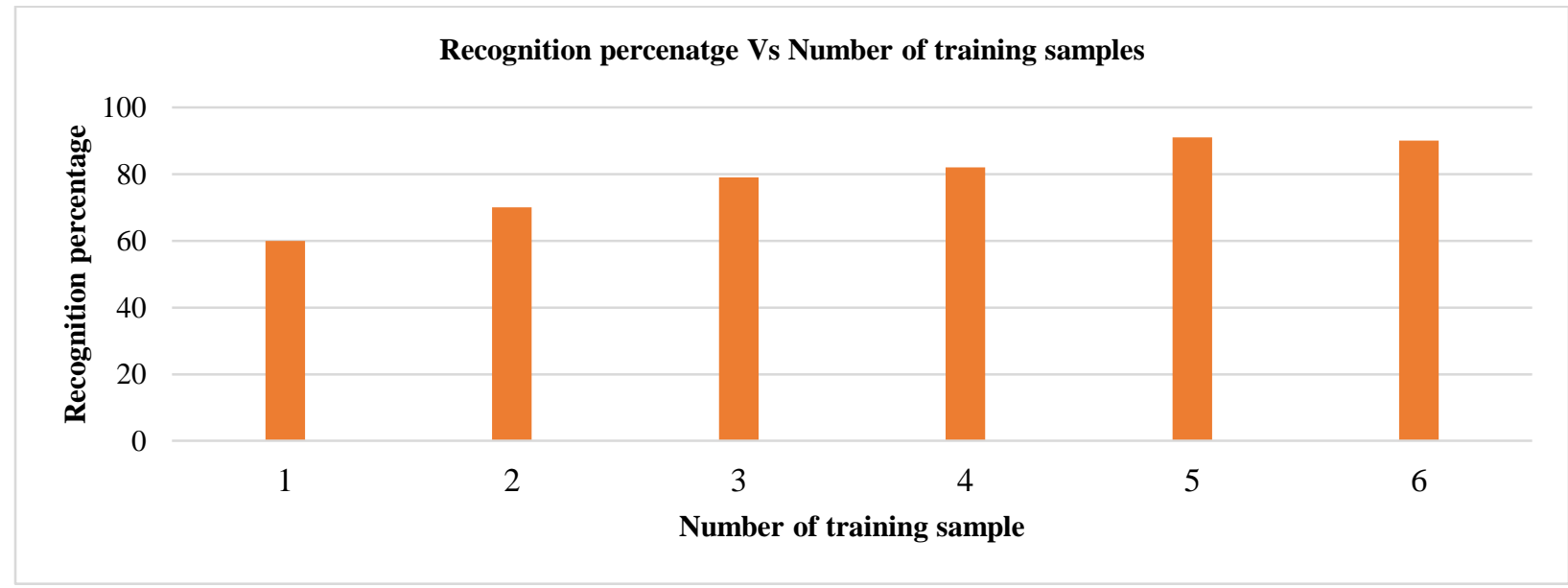

Figure 6 Recognition rate 


\section{Conclusion}

Our experiments employing a face database have shown that the proposed model are able to do a recognition rate of over $90 \%$, capturing 93 poses from swish head rotation is at intervals 90 degrees from the sample view. This model is easy and reliable with increasing recognition accuracy. For future works on the method, we plan to test the performance of the method on larger database with different shots.

\section{Acknowledgment}

None.

\section{Conflicts of interest}

The authors have no conflicts of interest to declare.

\section{References}

[1] Zhao W, Chellappa R, Phillips PJ, Rosenfeld A. Face recognition: a literature survey. ACM computing surveys (CSUR). 2003; 35(4):399-458.

[2] Phillips ML, Bullmore ET, Howard R, Woodruff PW, Wright IC, Williams SC, et al. Investigation of facial recognition memory and happy and sad facial expression perception: an fMRI study. Psychiatry Research: Neuroimaging. 1998; 83(3):127-38.

[3] Blackburn DM. Face Recognition 101: A. A Brief Primer Version. 2003; 2(07).

[4] Muruganantham S. Pose invariant face recognition-a new technique. International Journal of Computer Science Engineering and Information Technology Research. 2013; 3(3):135-40.

[5] Liau HF, Ang LM, Seng KP. A multiview face recognition system based on eigenface method. In 2008 International Symposium on Information Technology 2008 (pp. 1-5). IEEE.

[6] Maurya JP, Waoo AA, Patheja PS, Sharma S. A survey on face recognition techniques.2009; 5(2):4168.

[7] Lu Y, Zhou J, Yu S. A survey of face detection, extraction and recognition. Computing and Informatics. 2012; 22(2):163-95.

[8] Shamna P, Paul A, Tripti C. An exploratory survey on various face recognition methods using component analysis. International Journal of Advanced Research in Computer and Communication Engineering. 2013; 2(5):2081-6. 